\title{
Making love in the time of corona - considering relationships in lockdown
}

\section{Marieke Dewitte, Chantelle Otten and Lauren Walker}

Abstract | The COVID-19 pandemic and the resulting social changes that were required to slow the spread of severe acute respiratory syndrome coronavirus 2 (SARS-CoV2) have resulted in lockdowns across many countries and led to substantial numbers of people being quarantined. For single people, their opportunities to meet a partner were completely lost. For couples who lived apart, this meant that they were not able to see their partner for many months. However, by contrast, for cohabiting couples, lockdown meant that they were forced to spend $24 \mathrm{~h}$ a day with each other, and perhaps their children or housemates, for months at a time. As lockdowns have loosened around the world, the possibility of a second wave arises, and lockdowns are being reinstated in many regions. The prospect of potential long-term lockdowns means that adjusting to this new normal in relationships is an important consideration. In this Viewpoint, three specialists in sexology and psychology discuss the effects of lockdown on intimacy and consider how it can be considered an opportunity as well as an obstacle for making love in the time of corona.

SARS-CoV-2 can be transmitted by respiratory droplets and some early data have suggested that it might be present in semen. Assuming that sexual transmission could be possible, should this alter how partners approach intimacy?

Marieke Dewitte. There is no solid evidence that coronavirus disease 2019 (COVID-19) can be transmitted via genital and anal contact, but it will be passed on via kissing and physical touching, which are common practices during partnered sexual activity. Although engaging in sexual and intimate activities with partners who live in the same household is safe as long as none of them shows COVID-related symptoms, some people will refrain from all intimacy on principle, out of fear of getting or spreading the virus. This avoidance is unfortunate because physical touch is an essential part of sexual intimacy, constitutes a key determinant of emotional connectedness and can even be considered a necessity of life. That is, touch has a calming effect by decreasing levels of cortisol and increasing oxytocin, which is the primary hormone involved in social bonding and also facilitates sexual arousability ${ }^{1}$. The pandemic and the accompanying social mitigation measures have created a clear paradox between, on the one hand, deep fear of close contact with other people and, on the other hand, an intense longing for physical touch, in particular being hugged and cuddled, as a means of coping with distress and increasing feelings of interconnectedness.

Sex and relationships in COVID times have gained much media attention, including suggestions on how to limit contamination risks during sexual activity by using sex toys, webcam and phone sex, and mutual masturbation, for example. Even official authorities, such as the New York City government and the Australasian Society for HIV, Viral Hepatitis and Sexual Health Medicine, have proposed that partners explore sexual activities without direct physical contact, with masturbation being the safest option. Although masturbation does indeed limit the risk of contamination and has benefits in terms of stress relief, solitary sex can never replace partnered sex because it serves fundamentally different functions.
Sexual desire is not an inherent drive that suddenly pops up and needs to be released and gratified ${ }^{2,3}$. Sexual desire can arise from many different underlying motivations, with dyadic sexual pleasure and expression of relational intimacy being important motivators ${ }^{4}$.

Living conditions for many cohabiting couples have changed significantly in lockdown, with more time together, less alone time, and perhaps more time with their children. How might this affect intimacy?

M.D. Although both media and research efforts are directed towards generating means and averaged numbers on the effects of COVID-19 (measures) on sexual relationships, it is difficult to draw overall conclusions because many individual differences exist in how people react to (pandemic) stress, depending on specific individual, relational and contextual characteristics. Some people completely lose interest in sex; other people experience an increase in sexual desire and use sex as a coping mechanism to stay connected and relieve anxiety. Differences within and between couples might become more apparent during this COVID-19 crisis, and individual differences in the way people cope with stress and fear might increase the risk of sexual desire discrepancies within a couple.

Furthermore, the social mitigation measures pose specific challenges to different types of couples, including new couples who had to stop dating - at least in terms of face-to-face contact - or decided to move in together quickly and perhaps prematurely; young adolescent couples who experienced an abrupt disruption in their sexual and relational exploratory behaviour with a partner; couples in long-distance relationships who were suddenly forced to explore alternative means of staying intimately and sexually connected (such as remotely controlled sex toys); couples who just experienced a relationship break-up and were still adjusting to their new living situation, who were also suddenly deprived of social support; couples who were facing relationship difficulties and needed to interrupt therapeutic help or continue via teleconsultation; and couples in which one 
partner lives in residential care, who were suddenly not allowed any visits. How each of these couples will cope with pandemic stress depends on their personal and relational vulnerabilities and resilience; the presence of extra stressors that might exaggerate or escalate existing relational and sexual difficulties is clear. COVID-19 lockdown has meant that couples in distress need to work through a crisis on top of a crisis.

Without drawing general conclusions or ignoring intrapersonal and interpersonal differences in the meaning of pandemic stress for individuals and couples, high-stress situations and prolonged quarantine have been shown to induce symptoms of depression, post-traumatic stress, loneliness, confusion, anger, frustration, boredom, worry and health-related fear ${ }^{5}$. The sudden change in daily routine, limiting personal and work activities and spending more time at home increases anxiety and stress levels and might disrupt one's sense of self. That is, many people had to change and adapt to their roles, for example, from being focused on their career to being a full-time parent, with little or no opportunity to become accustomed to this change ${ }^{6}$.
Furthermore, the stress of being at home all the time and combining different roles has meant that some people have been able to invest less time in self-care, have experienced weight changes and, overall, feel less good about themselves, which result in them feeling sexually less attractive. These identity disruptions, together with reduced social contact and restricted engagement in valued activities or healthy forms of non-COVID-19-related behaviour, will be likely to induce distress, adding to existing fears about health and the socioeconomic aftermath of the COVID-19 crisis. This preoccupation with fearful thoughts can distract attention from sexually intimate cues, lowering sexual arousability and possibly even inducing sexual problems such as genital pain or erectile dysfunction ${ }^{7}$.

\section{Lauren Walker. When the lockdown} initially started, there were lots of jokes on social media and in my social network that with nothing else to do, people would be having a lot more sex! But the reality is that some people do not have access to partners because of distancing measures, and others have so much contact with their partner

\section{The contributors}

Marieke Dewitte is a clinical psychologist-sexologist who had her training in Belgium and the Netherlands and completed her PhD at Ghent University, Belgium. She currently works as Assistant Professor at the Department of Clinical Psychological Science of the University of Maastricht, Netherlands. She teaches several courses and workshops on sexual responding at the university and in post-academic sexology training. Her research involves psychophysiological studies on basic mechanisms of sexual functioning, gender differences, interpersonal dynamics, dyadic interactions between partners, and attachment in relation to sexual responding. The main focus of her research is on the sexual interaction between partners. Her clinical (research) focus is on female genital pain. She also worked as a sex therapist at Ghent University Hospital and now at Maastricht University Hospital.

Chantelle Otten holds a Masters in Science Medicine, Sexual Health/Psychosexual Therapy from the University of Sydney and is a European Federation of Sexology (EFS) and European Society of Sexual Medicine (ESSM) certified Psycho-Sexologist. Chantelle is passionate about empowering people to feel great about their sexual health, self-esteem, communication and education. A Melbourne local, Chantelle comes from a Dutch background. Having spent time living, studying and working in the Netherlands, Chantelle has grown up with the European mindset that talking about sex, pleasure and relationships does not have to be shameful or taboo. With a background in scientific research, sexual medicine and counselling, she believes that sexuality and self-esteem are an integral part of life, to which everyone is entitled. Good sexual health should always be enjoyable, pain free and without prejudice. Chantelle is the director of the Australian Institute of Sexology and Sexual Medicine, where she and her team of sexologists work to positively change the sexual lives of Australians, and she also uses social media to spread sexual empowerment to all.

Lauren Walker is a clinical psychologist with 10 years of experience in sexual medicine. She is an Adjunct Associate Professor and directs the Oncology Sexual Health Lab at the University of Calgary. She conducts research creating and evaluating sexual health resources for cancer patients. She has over 35 publications in the area of sexual health and cancer. She supervises research trainees and regularly speaks at local and national educational events for patients and for health-care providers. Dr Walker operates a private clinical psychology practice in the community in Calgary, AB, specializing in sexual health for patients and couples. She also uses Instagram to increase the accessibility of sexuality education — check out her account @drlaurenwalker.

that their relationship is strained such that sex may be the least of their interests. Add in the challenges of parenting small and even young adult children, and the result is limited time, energy and privacy to engage in sexual adventure. I have been having a lot of conversations with my patients about privacy: how to ask for privacy and lack of interruption from a partner or a roommate to ensure alone time for solitary sexual activities, or how to ensure privacy from children so that couples can engage in intimate activities. Notably, this does not just mean intercourse! Couples might also want time for massage, bubble baths, or just having uninterrupted adult conversation.

Justin Lehmiller's work suggests that couples are not having more sex during COVID-19, but that the sex they are having is more adventurous ${ }^{8}$. Thus, one approach might be that if you are able to set up some private time, get the children settled with snacks and a movie, or wake up before they get up in the morning, you might want to try something new. Crack out some sexual toys, find a blindfold, try a new position, or act out a sexual fantasy. If you are looking for ways to spice up your activities, you might consider asking a partner about their fantasies, or what is something new that they might be open to trying. Arguably though, we should not have to wait for a pandemic to explore this kind of growth in a sexual relationship.

M.D. Although sexual frequency can either increase or decrease in times of prolonged stress, the quality of and satisfaction with sex will generally decrease ${ }^{9}$. However, we have to be careful not to focus too much on the facilitating or inhibiting effect of pandemic stress on levels of sexual desire and frequency of sexual activity. Given that sexual desire unfolds in response to (adequate) sexual cues and does not arise spontaneously, the question is not whether stress has a direct impact on desire but rather whether it will interfere with the opportunities to create or search for sexual stimulation and the motivation to engage in sexual activities when initiated. Being absorbed by work-household conflicts or relational issues that have surfaced owing to couples having less privacy in the confined space of their home, the continuous time together, the confrontation with feelings of loneliness, lack of emotional support and relationship conflicts, and the lack of (social) distractions or occasions to escape in work or hobbies can reduce both the time and motivation to invest in sex. 
[] Is there a positive effect of sex to manage the stress of living in a pandemic - should we in fact encourage it (taking into account guidelines) as a benefit to mental health?

M.D. Some couples might well benefit from this crisis and experience the lockdown as an opportunity to reconnect and reinvent their sexual relationship by breaking routines, investing more time together, and actively working on their relational and/or sexual issues. Some people have reorganized their value system by incorporating a better work-life balance, building healthy food and sports habits that make them feel more attractive and, therefore, more responsive to sexual initiatives. Some couples are creatively seeking out new ways of experiencing relational intimacy, of rewriting their sexual script, and of prioritizing their own partner, also through having fewer opportunities to meet other people and engage in infidelity. In this context, the paradoxical effect of relational closeness on sexual desire is an important consideration. That is, when there are fewer opportunities for independent activities or time apart and when intimacy collapses into fusion, sexual desire might also be impeded because too much togetherness disrupts the balance between our need for closeness and our need for autonomy and separateness ${ }^{10}$. Variation and flexibility to adjust to changing life circumstances are an important determinant of mental health and well-being. Such flexibility, acceptance and openness to live a vital and valued life despite the COVID-19 pandemic will promote relational growth instead of post-traumatic stress ${ }^{7}$.

Chantelle Otten. Sex has always been a great stress reliever and mood booster; experiencing pleasure and intimacy just makes us feel good! At the moment it feels like we should be utilizing anything we can to lift our spirits, including the more erotic options at our disposal. Sex can help us to feel more in tune with our partner and ourselves; it can anchor us to the present, making us feel stable and secure in the now (which is a rarity at the moment). People always say that physical movement, such as the gym or walks, are great for our mental health and are things we should be maintaining, however we can, in lockdown. Thus, sex, as a pretty energetic activity that gets our hearts pumping, blood flowing and pleasure senses tingling, is a no-brainer to improving our mental health.
L.W. The bottom line is that during times of stress, everyone reacts differently in terms of their interest in sex. We are seeing vast impacts of COVID-19 on the lives of individuals, including health anxiety, financial uncertainty, threat to safety, social isolation, and increased demands associated with full-time caring for children, to name just a few. For some people, any one of these factors can be a significant 'libido killer'. For others, sex (alone or partnered) can be a source of managing stress ${ }^{11}$, one that allows empowerment to feel pleasure, control during a time of uncertainty, energy release, and bonding with someone we love. Whatever level of desire you are feeling or not feeling is fine - do not add more stress to your life by thinking your level of desire is too high or too low; there is no 'right' level of sexual desire.

Another thing to consider is how you are showing love to yourself during this time. Self-care has never been more important than it is now. If one of the ways you can care for yourself is to be sexual (whether this is alone or in a safe partnered scenario), then give yourself permission to do so.

If you are finding that sex helps you to manage stress better, you might appreciate some of the health benefits of regular sex, perhaps particularly relevant at the moment. Research suggests a link between orgasms from penile-vaginal intercourse and reduced blood pressure ${ }^{12}$, as well as between sexual activity and pain control ${ }^{13}$, improved immune function ${ }^{14}$ and improved cognitive capacity in older adults ${ }^{15}$. Pleasurable sexual touch is also associated with improved genital health. The research on these matters is cross-sectional and, therefore, does not provide the ability to infer causal relationships (that is, healthier people might just be more likely to have sex), but it seems unlikely that positive sexual experiences would have negative effects on health and well-being.

M.D. The media might also have had a role in how couples cope with pandemic stress by directing and shaping the perception and expectation of their sexual relationship. Media coverage has amplified the psychosocial effects of this pandemic by communicating feelings of uncertainty or even panic, which also applies to messages about the potential positive or negative effects of the lockdown on sex and relationships, thereby creating new norms and performance demand or providing excuses, which could induce or aggravate relational and sexual problems. Depending on which information they choose to

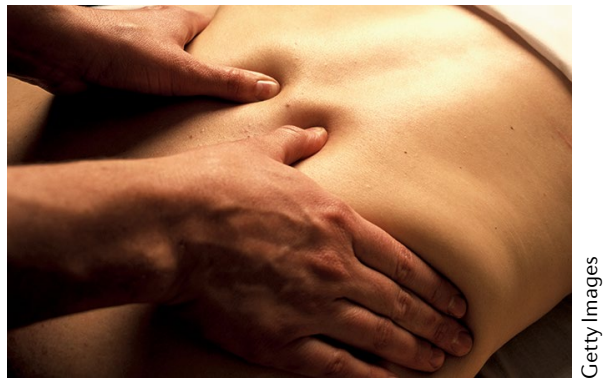

attend to, couples might start to act in a way that confirms these positive or negative messages about COVID-19 and (sexual) relationships.

What about couples who do not live together and are now separated? How can they maintain intimacy and their relationship?

C.O. All couples, and individuals, need to be adhering to the isolation rules of their current state, which means that some couples might be separated from each other at the moment or might have experienced a long period of separation that is now coming to an end, at least for the time being. In these situations, intimacy is possible! We just need to embrace the creative side of our brains. People who have experienced long-distance relationships will attest that, although they can initially be a challenge, separation can actually create more openness and intimacy with our partners. In the current digital age, countless options are available to stay erotically connected to our partner: phone sex, sexting, video call sex, swapping pictures, videos, erotic poems or stories, to name but a few! Lockdown could actually be a great time to practice your dirty talk, or talk through a fantasy with your partner (without any stress of having to actually do it!). However, it is not all just about sex (shocking, I know) and couples need to maintain intimacy in other ways. Maybe it means you call each other and watch the same movie together, have a video call date, send each other letters describing all the things you love about each other. Whatever things you would usually do with your partner that make you feel connected, think about how you can recreate that feeling from afar.

L.W. The good news for couples who are separated is that we live in the digital age. Just as workers across the world are adapting their practice to work remotely, couples can also engage in sex remotely. Of course, there are some considerations. Some of which might be less of a concern if you have 


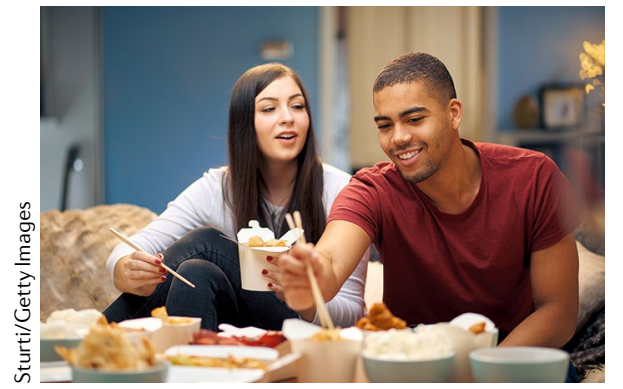

a trusting relationship with your partner, or if this is something you already have experience doing with this partner. First, always start with consent. Do not assume just because your partner is flirting that they are interested in more sexually explicit conversation. Ask directly, for example, "would you like to talk more about what you might fantasize about"? Or ask if they would be interested in seeing a picture of you, and also ask what kind of picture they would like (clothed versus naked, full body versus genitals only). Second, ask if now is a good time. Just because your late-night direct message was met with a reciprocal response last week, does not mean that the same time this week will be a good time for your partner: privacy, availability, need for sleep, sexual interest and so on all vary from day to day. Before engaging in these activities, talk to your partner about expectations around saving or not saving photos or screenshotting content. You might want to consider using an encrypted platform for video or text-based chat. Another consideration may be to use an account that is not linked to your name or phone number. Finally, it is safest to not include your face in photos or, even safer, avoid text-based communication altogether and consider just talking on the phone! Also good sex does not always require a partner! Remember you are also fully capable of pleasuring yourself.

\section{What do you say to people who assume that sexuality is not a priority during a pandemic?}

M.D. The apparent psychosocial consequences of this pandemic have raised general awareness about the importance of mental health and the necessity to invest more resources in prevention and treatment. People cannot cope well with events that induce feelings of unpredictability and uncontrollability, which increases the risk of developing mental health problems or aggravating (and inducing new) psychiatric symptoms in people with pre-existing problems. It has even been argued that mental problems will become a 'secondary' epidemic, posing new challenges. The importance of sexual and relational variables as a key determinant of mental health and quality of life, however, has not (yet) captured sufficient attention in policymaking. Often, sexual and relational problems are considered as 'secondary' problems, thereby disregarding the mutual influence between sex, relationships and mental health, as well as the impact of relational stressors specific to this pandemic.

L.W. The bulk of my research has focused on sexuality in the context of cancer, and I receive this kind of comment a lot from cancer-care providers. In oncology, providers often assume that the patient's focus is (or should be) on life-saving treatments, rather than on sexuality. In a context where the cancer treatments themselves are associated with a multitude of negative effects on sexuality, this opinion is concerning ${ }^{16}$. The same can be said for the pandemic, where the health-care system is focused on preventing COVID-19 infections, to 'flatten the curve' and to decrease the burden of active cases on the system. However, individuals with sexual concerns are still suffering and still in need of care. They should not be asked to wait 3-6 months to address their concerns after the pandemic settles. The longer sexual difficulties go unaddressed, the more entrenched they tend to become, and often do not often resolve on their own ${ }^{17}$. Individuals who consider sexuality to be an important component of maintaining good quality of life, should also be encouraged and supported to continue to maintain satisfying sexual relationships and activities as best they can, while of course ensuring safety. If we as providers ignore sexuality in the context of the pandemic, assuming that other, more pressing issues should be the focus, these patients will assume that sexuality is not a valid concern to bring up to their doctor. Thus, even in times where highly pressing concerns might be at the forefront of our minds as providers, we should not neglect including questions about sexuality in our routine assessments. The comments I often hear from providers suggest hesitance to ask about sexuality for fear of embarrassing the patient by bringing up something that is not on their minds, or that could make them uncomfortable. Instead, consider phrasing the inquiry in a ubiquitous style such as, "Stressful times like these can often have a negative impact on sexuality, is that a concern for you at all?" whereby the patient either feels that their concern is normalized and safe to talk about, or if they do not want to entertain further discussion they can simply report "No". They might not take you up on the offer to talk further, but you have already begun the work to support your patients in letting them know that you are open to discussions about sexuality should the need arise in the future.

\section{Is there a role for telehealth in sexual medicine during the COVID-19 pandemic and beyond?}

M.D. The social mitigation measures have led many health professionals to switch to teleconsultation, offering their patients therapy and support via video calls and other online platforms. Some health-care providers have found that this type of consultation puts a barrier between human contact and social interaction and consider it vastly unsuited to a profession such as sex therapy, which is built on trust, close contact and the observation of non-verbal cues. Given that many individuals and couples with sexual problems already feel hesitant to seek help, video calls with someone whom they have not yet grown to trust might increase the barrier to start or even continue therapy. On the other hand, studies on internet therapy have shown that online help is more cost-effective and readily accessible than traditional treatment modalities, thereby helping to overcome common barriers to seeking help such as shame, difficulties with face-to-face communication about sex, time limits, costs or geographical distance ${ }^{18}$. The current situation could, therefore, be used to scale up telemedicine and expand its applications beyond providing online relationship and sex therapy towards including services for medication, abortion, contraception, prevention of sexually transmitted infections (STIs) and trauma-informed care for managing gender-based violence. However, careful attention must be paid to guaranteeing the quality of online interventions by preventing people from offering online help without a license - as sex therapy is not a recognized profession in almost all European countries, online interventions could make it easier for people to start offering unprofessional and low-quality care.

C.O. Definitely! I have seen many clients online during the pandemic, and telehealth is actually a great option in general for people who are unable to physically attend a session. We have such amazing online options these days that enable great health care from a distance. Although I love seeing 
my clients in person, I would never want to remove the option of care completely for them just because we cannot physically be in the same room together. Sexual health is important all the time.

L.W. Absolutely. Telehealth is a great option - it is how I have been seeing all of my patients. The same conversation about privacy applies here. A couple of tips: first, use headphones - this helps to reduce the risk of being overheard such that only half of the conversation could potentially be heard. Second, do not be afraid of judgement from your health-care provider; for example, a messy house or children asking for snacks or other things. Providers are there to support you - not to judge you (and chances are their house looks the same!). Third, find a private corner in the house: a closet, a bedroom, a garage, a car, or even go for a walk if your cellular service can support it. Finally, do not just wait the pandemic out to address your sexual concerns - there are ways to make remote meetings for sexual health care work. Your therapist or provider can also help you to troubleshoot barriers that come up for you.

Some people might be experiencing negative sex-related or intimacy-related consequences of lockdown - how can these be approached and managed?

L.W. Distancing protocol might actually present an unprecedented opportunity for several things. First, consider getting to know yourself better! If you are not able to access a sexual partner because of the distancing protocol, there is no need to stop being sexual. I work with so many patients who have barriers to engaging in masturbation or sexual self-exploration, such as guilt, shame, self-judgement, lack of confidence and even just a lack of know-how. Provided that you are open to the idea, but just do not know where to start, you might be surprised how much you can find if you are brave enough to type "masturbation ideas" into Google. In addition, if sexual interest is still high, solo sexual activity can still be a source of pleasure, enjoyment, stress release and self-care, during a time where these experiences may be limited by the pandemic.

If self-exploration is not your thing, you might consider how you can cultivate a sense of sexuality, even in the absence of explicit sexual expression. Sexuality and sex are not the same thing. Consider how you might express your sexuality in other ways: this might include the clothes you wear, the music you listen to, the food you eat, the wine you drink, the shows you watch on TV or the accounts you follow on Instagram. Sexuality does not always have to be about genitals, it can also be about mindset and personality too.

Finally, if you and your partner are not really feeling it, why not take the sexual agenda off the table? Consider pressing pause on goal-oriented activities like intercourse or seeking orgasms and try out some sensual full body massage. Share pleasure with each other, in the form of physical touch, without the pressure of sexual performance.

M.D. In addition to exploring the potential negative effects of stress on sexual activities and relationship functioning, which has attracted most of the media and research attention, another important effect of the coronavirus crisis that has tended to be overlooked is the reinforcement of existing disparities in terms of sexual and reproductive health and gender-based violence and stigma. Some groups are more vulnerable to the pandemic mitigation measures, including young people, women, LGBTQI-identifying people, refugees and migrants, the poor and uninsured, ethnic minorities, and people living with HIV. Those people whose human rights are least protected are likely to experience unique difficulties from COVID-19, which illustrates the need for a justice framework to monitor and address the inequitable gender, health and social effects of COVID-19 (REF. ${ }^{19}$ ). When looking at these vulnerable groups from a syndemic perspective, multiple health challenges can be seen to produce an increased burden of disease $\mathrm{e}^{20}$. We need to consider effects such as increased sexual risk behaviours, disrupted dating strategies, reduced access to and opportunities to have sex, increased substance use, and reduced access to STI testing facilities, STI treatment and HIV care. People with HIV might be immunocompromised, potentially increasing the risk associated with COVID-19 infection. When pre-exposure prophylaxis and HIV testing and care are interrupted, the sexual health of men who have sex with men and people living with HIV is compromised. To avert increased HIV and STI incidence, steps need to be taken to improve access to sexual health services, such as increasing the use of telehealth for pre-exposure prophylaxis and HIV care and mailed self-collection of specimens for HIV and STI testing ${ }^{20}$.

Women are another vulnerable group ${ }^{21}$. Although fewer women die from COVID-19

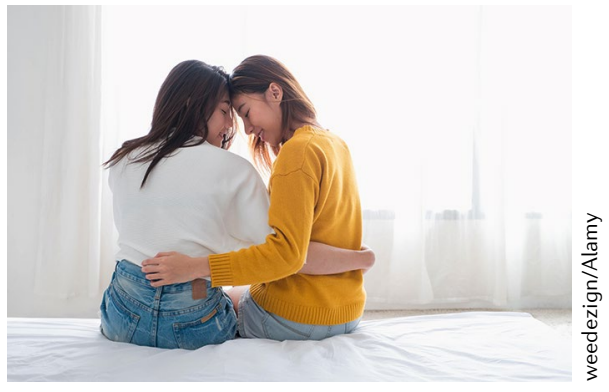

than men, the risk of experiencing psychosocial and systemic consequences is increased for women compared with men ${ }^{21}$. Pregnant women are at risk because routine prenatal care appointments have been interrupted, they need to deliver without the partner being present, and might lack practical and social support, leaving them vulnerable during the first weeks at home with a newborn. Furthermore, difficulties in obtaining regular contraceptive methods or emergency contraceptives and restrictions on certain medical procedures such as abortion increase the risk of unintended pregnancy. Women more frequently than men act as the primary caregivers within a household, increasing the burden and stress they experience when combining work and children. The risk of domestic abuse and violence, which is most often directed towards women, is also increased and fewer social interactions also imply less accountability for perpetrators and provides fewer opportunities for others to intervene. Finally, women constitute a large proportion of frontline health-care professionals, facing a higher risk of infection, morbidity and death as a result of their profession. These health workers might also experience considerable stigma and discrimination because of their increased risk of being contaminated.

Other groups are also unduly affected by social pressure and stigma during this pandemic. LGTBQI-identified individuals might now experience increased obstacles during their coming-out process and increased feelings of non-belonging, as gay men are sometimes associated with having promiscuous sex and, therefore, are (wrongly) assumed to carry a high risk of becoming exposed to the virus ${ }^{19,21}$. Transgender people could experience social and psychological difficulties when their medical transition is put on hold during the pandemic. Sex workers are considered by some to be super-spreaders of the virus, thereby increasing the stigma that is already associated with this profession. Finally, we might consider the stigma associated with people who are not able to respect the 


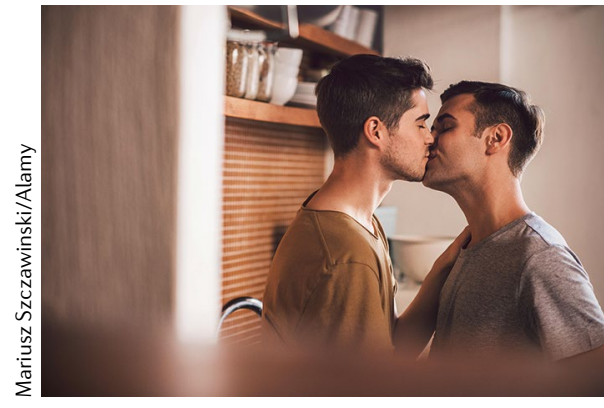

social mitigation measures, such as single people who feel guilty and ashamed about their fundamental need for physical touch and sexual interaction.

As a field focusing on sexual and reproductive health, we cannot ignore these inequalities and we must raise our voice to make these issues count in the political field.

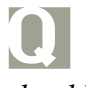

Finally, can you make any recommendations for how people should approach, maintain and potentially even improve their sex lives during this time?

C.O. First, be kind to yourselves. In these times of unrest and uncertainty, it is OK not to be feeling your most sexy and sexual. You might also have had to adjust to children being home $24 / 7$, or working at home with your partner and maybe spending almost too much time together. So allow yourself time to adjust and settle, but let us not forget what a great stress reliever sex can be. If you find yourself with a lot of time on your hands, take it as an opportunity! Try that complicated position you have always wanted to try, if you can, or maybe take a good hour (or 4!) to sensually enjoy yourself or your partner. View it as the time to be really sexually free in your wants and desires.

M.D. Instead of counting and complaining about the number of times partners have had sex during this period, it might be more useful to focus on renegotiating the meaning of sex and revaluing the importance of sexual intimacy and physical touch as a means of reducing stress. Moments of crisis provide opportunities to change course, reconnect, introduce a new perspective, revalue the partner and revive relational dynamics that have been there before and fuelled sexual attraction. Having sex is about making an effort, planning and creating opportunities, thereby challenging the myth of spontaneous sex that puts undue pressure on many couples. The past quarantine period, and maybe those that are still ahead, might leave us with more or fewer occasions to have sex in terms of time and resources. Couples should be aware of the benefits of having sex during periods of stress without trying to meet any new standards or external pressure. There is neither an ideal set point nor an ideal sexual script to follow. Instead, couples can use this disruption of daily routines to broaden their sexual script defining sex as more than only penetration, thinking outside the box, initiating open communication about sexual likes and dislikes and introducing sexual pleasure and play instead of sexual duty. Our daily context can be considered as a form of foreplay that prepares for sexual intimacy, indicating the importance of investing more in relationship quality markers such as support, constructive conflict resolution, attachment and partner responsiveness. In addition to increasing the feeling of interconnectedness, it is also important to leave each other some space and keep a good balance between togetherness and separateness. Partners can start engaging in new activities, both individually and as a couple, in order to introduce an element of novelty and mystery, thereby seeing their partner through a new gaze, which could eventually increase sexual desire and attractiveness. However, during this crisis, some couples have been confronted with the emptiness, emotional distance and lack of connection in their relationships that might have been masked for many years. COVID-19 is not an excuse to look away or ignore existing problems, but it is important to keep in mind that these are exceptional circumstances that put exceptional pressure on the relationship, which might motivate people to be more mild, tolerant and accepting towards each other. In such times, it is recommended that people do not make hasty decisions about separating; however, those who do should not feel judged when making this choice, especially when the relational climate is damaging and unhealthy to both partners and children.

\section{L.W. Couples and families might have a} unique opportunity here, while their social bubble is reduced, to really focus on each other. Your calendars are not full of social obligations, so instead create some rituals and protected time for special activities: family movie night, picnics and campouts in the backyard, or a spa day "Chez Vous Spa". A little imagination and you might even transform your living room into the bustling cobblestone streets of Florence, Italy! Open a bottle of Chianti and dine on spaghetti pomodoro with Vivaldi playing lightly in the background. These opportunities enable you to act out a fantasy, even in a world where we are limited by what we can actually do in reality (because of the pandemic or otherwise). See if you can work with your partner or your family members to create these unique opportunities for ritual and set these experiences apart from the grind of your daily routine. Marieke Dewitte ${ }^{\boxplus}$ Chantelle Otten $^{2 凶}$ and
Lauren Walker $^{\circledR}$

${ }^{1}$ Department of Clinical Psychological Science, Maastricht University, Maastricht, Netherlands.

${ }^{2}$ The Australian Institute of Sexology and Sexual Medicine, Melbourne, Victoria, Australia.

${ }^{3}$ Division of PsychoOncology, Departments of Oncology \& Psychology, University of Calgary, Calgary, Alberta, Canada.

凶e-mail:marieke.dewitte@maastrichtuniversity.nl; chantelle@chantelleotten.com; Imwalker@ucalgary.ca https://doi.org/10.1038/s41585-020-0365-1

Published online 20 August 2020

1. Uvnäs-Moberg, K., Handlin, L. \& Petersson, M. Self-soothing behaviors with particular reference to oxytocin release induced by non-noxious sensory stimulation. Front. Psychol. 5, 1529 (2014).

2. Meston, C. M. \& Buss, D. M. Why humans have sex Arch. Sex. Behav. 36, 477-507 (2007).

3. Both, S., Everaerd, W. \& Laan, E. in The Psychophysiology of Sex (ed Janssen E.) (Indiana University Press, 2017).

4. Toates, F. An integrative theoretical framework for understanding sexual motivation, arousal, and behavior. J. Sex. Res. 46, 168-193 (2009).

5. Brooks, S. K. et al. The psychological impact of quarantine and how to reduce it: rapid review of the evidence. Lancet 395, 912-920 (2020).

6. Presti, G., McHugh, L., Gloster, A., Karekla, M. \& Hayes, S. C. The dynamics of fear at the time of COVID-19: a contextual behavioral science perspective. Clin. Neuropsychol. 17, 65-71 (2020)

7. Barlow, D. H. Causes of sexual dysfunction: the role of anxiety and cognitive interference. J. Consult. Clin. Psychol. 54, 140-148 (1986).

8. Lehmiller, J. J., Garcia, J. R., Gesselman, A. N. \& Mark, K. M. Less sex, but more sexual diversity: changes in sexual behavior during the COVID-19 coronavirus pandemic. Leis. Sci. 17, 1225-1228 (2020).

9. Yuksel, B. \& Ozgor, F. Effect of the COVID-19 pandemic on female sexual behavior. Int. J. Gynecol. Obstet. 150, 98-102 (2020).

10. Schnarch, D. M. Passionate Marriage: Sex, Love and Intimacy in Committed Relationships (Norton, 1997).

11. Ditzen, B. et al. Intimacy as related to cortisol reactivity and recovery in couples undergoing psychosocial distress. Psychosom. Med. 81, 16-25 (2019).

12. Brody, $\mathrm{S}$. Blood pressure reactivity to stress is better for people who recently had penile-vaginal intercourse than for people who had other or no sexual activity. Biol. Psychol. 71, 214-222 (2006).

13. Hambach, A., Evers, S., Summ, O., Husstedt, I. W. $\&$ Frese, A. The impact of sexual activity on idiopathic headaches: an observational study. The impact of sexual activity on idiopathic headaches: an observational study. Cephalgia 33, 384-389 (2013).

14. Charnetski, C. J. \& Brennan, F. X. Sexual frequency and salivary immunoglobulin A (IgA). Psychol. Rep. 94, 839-844 (2004)

15. Wright, H., Jenks, R. A. \& Demeyere, N. Frequent sexual activity predicts specific cognitive abilities in older adults. J. Gerontol. Ser. B 74, 47-51 (2019).

16. Robinson, J. W., Lounsberry, J. \& Walker, L. M. Communicating About Sexuality in Cancer Care, Handbook of Communication in Oncology and Palliative Care. 2nd Eds (Oxford University Press, 2017).

17. Schover, L. R. et al. Sexual dysfunction and infertility as late effects of cancer treatment. EJC Suppl. 12, 41-53 (2014). 
18. Barak, A., Klein, B. \& Proudfoot, J. G. Defining internet supported therapeutic interventions. Ann. Behav. Med. 38, 4-17 (2009)

19. Hall, K. S. et al. Centring sexual and reproductive health and justice in the global COVID-19 response. Lancet 395, 1175-1177 (2020).

20. Shiau, S., Krause, K. D. Valera, P., Swaminathan, S. $\&$ Halkitis, P. N. The burden of COVID-19 in people living with HIV: a syndemic perspective. AIDS Behav. 24, 2244-2249 (2020)
21. Gausman, J. \& Langer, A. Sex and gender disparities in the COVID-19 pandemic. J. Womens Health 29 465-466 (2020)

Competing interests

The authors declare no competing interests.

Publisher's note

Springer Nature remains neutral with regard to jurisdictional claims in published maps and institutional affiliations.

\section{RELATED LINKS}

Australasian Society for HIV, Viral Hepatitis and Sexual Health Medicine: https://ashm.org.au/covid-19/clinical-care/

harm-minimisation-approaches-to-casual-sex/

NYC Government: https://www1.nyc.gov/assets/doh/

downloads/pdf/imm/covid-sex-guidance.pdf?utm_

source=morning_brew

(C) Springer Nature Limited 2020, corrected publication 2020 